\title{
Artificial Fish Swarm Algorithm-Assisted and Receive-Diversity Aided Multi-user Detection for MC-CDMA Systems
}

\author{
Zhicheng Dong, Wei Xiao \& Xiping Zhang \\ Electronic information engineering department \\ School of Engineering, Tibet University, Lhasa 850000, China \\ Tel: 86-817-288-4617Ｅ-mail: dongzc666@163.com
}

\begin{abstract}
Artificial fish swarm algorithm (AFSA) assisted multi-user detection (MUD) is proposed for the receive-antenna-diversity-aided multi-carrier code-division multiple-access (MC-CDMA) systems in frequency selective fading channel. Due to the receive-diversity, the signals received at the different antennas are faded independently, resulting in an independent objective function for each antenna. To resolve the multi-objective dilemma when choosing one signal estimation for multiple receive antenna-branches, the individuals associated with the AFSA are selected based on the concept of Pareto optimality, which uses the information from the antennas independently. Simulation results showed that: with the same computation complexity, the strategy has much better bit error rate (BER) performance than the convention one. Comparisons with the conventional multiuser detector and the decorrelator verified the effectiveness of the proposed scheme.
\end{abstract}

Keywords: Antenna-diversity, MC-CDMA, Artificial fish swarm algorithm, MUD, Pareto optimal

\section{Introduction}

Recently, the multi-carrier code-division multiple access (MC-CDMA) based on the combination of OFDM (Orthogonal Frequency Division Multiplexing) technique and conventional CDMA has attracted great interests in both practical and theoretical studies of modern mobile communications because of its high spectral efficiency and high data rate transmission(Abrao, T.; Ciriaco, F.; de Oliveira, L.D.; Jeszensky, P.J.E. 2006)(B. Steiner. 1997). Due to multiple users face serious multi-access interference (MAI) in the uplink and limit the capacity, the optimum multi-user detector (OMD) proposed by Verdu(C. Ergun, K. Hacioglu. 2000) in 1986. But the computation complexity of this detector increases exponentially with the number of users and make it impractical for an actual system. Consequently, the major portion of the scholars focus on suboptimal detectors, which are near-far resistant, have reasonable computational complexity, and approach the performance of OMD.

The mathematical model of MUD can be considered as a combinatorial optimization problem, which is also a NP-complete problem. Genetic Algorithm(GA) and Particle Swarm Optimization(PSO) can solve these problems effectively, so many scholars proposed the GA-based multi-user detection (GAMUD) and the PSO-based multi-user detection (PSOMUD)(D.E. Goldberg. 1989)(E. Zitzler, L. Thiele. 1999)(G. Syswerda. 1989)(Jianmei Xiao; Xiaoming Zheng; Xihuai Wang; Youfang Huang, 2006).

Artificial fish Swarm Algorithm (AFSA) is a new kind of intelligence optimization algorithm, which has some advantages that GA and PSO do not have. Multi-user detection based on AFSA has been proposed (K. Yen, L. Hanzo. 2003) and show that have better performance than GAMUD and PSOMUD.

In the receive diversity aided systems, the antennas are assumed to be sufficiently separated such that the received signals at the antennas are faded independently, resulting in an independent log-likelihood function (LLF) for each antenna. This poses a problem of multi-objective optimization due to the fact that while a specific signal estimation may be deemed optimum on the basis of the LLF of one antenna, the same estimation may not necessarily be deemed optimum in terms of the LLF of the other antenna. To independently use the fading information of different antennas, Yen and Hanzo (S. Verdu. 1986) proposed a genetic algorithm (GA) based approach which takes the Pareto optimality (U. Fawer, B. Aazhang. 1995) into the consideration. However, many researches show that AFSA approach not only has better performance than the GA approach but also has lower computational complexity (Yang Yu, Ya-fei Tian, Zhi-feng Yin. 2005).In order to resolve this multi-objective dilemma in a more effective and less complex way, this letter 
proposes an AFSA based MUD with Pareto optimal.

This paper is organized as follows. Section II describes the synchronous MC-CDMA system communicating over frequency-selective fading channel using some antennas. Section III describes the AFSA used to implement our proposed detector in conjunction with diversity reception, and analyses complexity issues. Our simulation results are presented in Section IV, while Section V concludes the letter.

\section{System Model}

We consider the uplink of the synchronous MC-CDMA systems (B. Steiner. 1997), where the base-station receiver is equipped with $\mathrm{M}$ far-spaced receive antennas ,shown in Fig.1,the sub-carriers and the length of the frequency domain spreading code is $\mathrm{N}$ and $\mathrm{K}$ synchronous users are accessed simultaneously .When considering the kth user, the information symbol $b_{k}(t)$ emitted by the kth user is multiplied with the frequency domain spreading code

$\left.C_{k}=1 / \sqrt{M} c_{k}(1), . . c_{k}(N)\right]^{T}$, where $c_{k}(n)= \pm 1$, with $(\square)^{T}$ denoting transpose; the path gain of user $\mathrm{k}$ from it's transmit antenna to the mth receive antenna is denoted as $\mathrm{h}_{(k, m)}, \mathbf{H}_{k, m}=\mathbf{F h}_{(k, m)}$ where $\mathbf{F}$ denotes the discrete Fourier transform (DFT). $\sqrt{A_{k}}$ denoted the received energy for the kth user.

The signal vector received at the mth receive antenna can be expressed in the following form

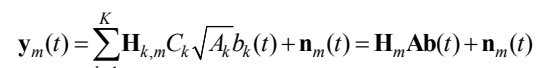

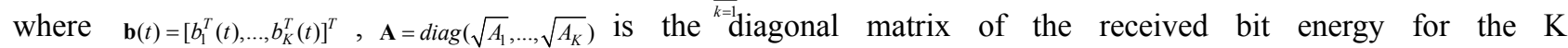
users. $\mathbf{H}_{m}=\left[\mathbf{H}_{1, m} \square C_{1}, \ldots, \mathbf{H}_{K, m} \square C_{K}\right]$, where $\square$ denoted multiplying element by element. where $\mathbf{n}_{m}(t)$ is the noise part received at the mth antenna, is the zero-mean complex additive white Gaussian noise (AWGN) with independent real and imaginary components, each having a double-sided power spectral density of $N_{0} / 2$.

At the receiver-side, the MLD detects all users' data to jointly minimize the effects of MAI (Y. Du, K.T. Chan, 2003). For the mth antenna, the log-likelihood function (LLF) is defined as

$$
\Lambda_{m}(b)=2 \operatorname{Re}\left\{b^{H} H_{m}^{H} y_{m}\right\}-b^{H} H_{m}^{H} H_{m} b
$$

The decision rule for the MLD associated with the mth antenna is:

$$
\hat{b_{m}}=\arg \left\{\max _{b}\left[\Lambda_{m}(b)\right]\right\}
$$

Since the channel fading of different receive antennas are independent, normally there exists $\Lambda_{m}(b) \neq \Lambda_{m^{\prime}}(b)$ where, $\left(m \neq m^{\prime}\right)$, When there exists deep fading in some antennas, the estimations of the transmitted signal corresponding to different antennas are not coincident, ie.

$$
\arg \left\{\max _{b}\left[\Lambda_{m}(b)\right]\right\}=\hat{b} \neq \arg \left\{\max _{b}\left[\Lambda_{m}(b)\right]\right\}
$$

Nevertheless, for the MLD, the LLF corresponding to different antennas are combined according to (Ying Zhao; Junli Zheng, 2004)

$$
\Lambda(b)=\sum^{M} \Lambda_{m}(b)
$$

The decision rule is then to find the signal estimation thät maximizes (5).

\section{AFSA-Based Multi-user Detection with Diversity Reception}

AFSA performs a parallel search in the solution space to find the optimum solution by simulating the behavior of fish including searching food, congregate and follow (Yang Yu, Ya-fei Tian, Zhi-feng Yin. 2005). In other words, this algorithm searches the optimum solution based on the cooperation and competition of the fish individuals .In this letter, we will employ AFSA in order to detect the estimated transmitted bit vector, shown in Fig .2.

AFSA commence their search for the optimum $\mathrm{K}$ bit state at the so-called $\mathrm{y}=0$ th generation by randomly creating $\mathrm{P}$ legitimate $\mathrm{K}$ bit state, where the pth artificial fish (AF) is expressed here as $\hat{\mathbf{b}}_{p}^{y}=\left[\hat{b}_{p, 1}^{y}, \hat{b}_{p, 2}^{y}, \ldots, \hat{b}_{p, K}^{y}\right]$.

\subsection{The behavior of selection}

Generally, in convention AFSA-MUD, the new AF swarm will been all selected for exploitation in the subsequent generation. In this letter, T AFs will been selected from the school, where $2 \leq T<P$, then produced a new population of offspring using the so-called uniform crossover(Z. Li, M. J. Juntti, M. Latva-aho, 2005) process.

In the convention strategy, each $\mathrm{K}$ bit $\mathrm{AF}$ is associated with a fitness value denoted as $f\left(b_{p}\right)=\Lambda\left(b_{p}\right)$, which is a function of the LLF of (5)(S. Verdu. 1986). AFs having the T highest fitness values in the population, where $2 \leq T<P$, are then selected and placed in the mating pool.

The second AF-selection strategy of the AFSA -assisted multi-user detection is based on the concept of the so-called Pareto optimality(U. Fawer, B. Aazhang. 1995). This strategy favors the so-called non-dominated AF and ignores the so-called dominated AF. Here, the pth $\mathrm{K}$ bit $\mathrm{AF}$ is associated with $\mathrm{M}+1$ fitness values denoted as $f\left(b_{p}\right)=\left[\Lambda_{1}\left(b_{p}\right), \ldots \Lambda_{M}\left(b_{p}\right), \Lambda\left(b_{p}\right)\right]$, where the first M fitness values are functions of the LLF of (2), while the last fitness value 
is a function of the LLF of (5). Then the ith $\mathrm{K}$ bit AF is considered to be dominated by the jth AF iff (U. Fawer, B. Aazhang. 1995)

$$
\begin{aligned}
& \forall m \in\{1, \ldots, M\}: \Lambda_{m}\left(b_{j}\right) \geq \Lambda_{m}\left(b_{i}\right) \\
& \wedge \exists m \in\{1, \ldots, M\}: \Lambda_{m}\left(b_{j}\right)>\Lambda_{m}\left(b_{i}\right)
\end{aligned}
$$

If an $\mathrm{AF}$ is not dominated in the sense of (6) by any other $\mathrm{K}$ bit AFs in the population, then by definition it is considered to be non-dominated. According to our second AF-selection strategy, all the non-dominated K bit AFs are selected and placed in the mating pool. Hence, the value of $\mathrm{T}$ in this case is not fixed, since it depends on the number of non-dominated AFs. If there is only one non-dominated $\mathrm{AF}$ in the current population, the next non-dominated AFs will be selected, so that there will be more than one AF in the mating pool.

\subsection{The behavior of crossover}

Two K bit AFs in the mating pool are then selected as parents based on their corresponding figure of merit in (5). The antipodal bits of the $\mathrm{K}$ bit parent vectors are then exchanged using uniform crossover(Z. Li, M. J. Juntti, M. Latva-aho, $2005)$ process, in order to produce two $\mathrm{K}$ bit offspring. The selection of $\mathrm{K}$ bit parents from the mating pool is repeated, until a new population of P offspring is produced(Z. Li, M. J. Juntti, M. Latva-aho, 2005).

\subsection{The behavior of congregation}

The new population of $\mathrm{P}$ offspring updates their state by the behavior of searching food, congregate and follow .According to the character of the problem, the AF evaluates the environment at present, and then selects an appropriate behavior. In the letter, the AF executes the behavior of congregate first, if its state cannot be improved, then executes the behavior of searching food(Yang Yu, Ya-fei Tian, Zhi-feng Yin, 2005).

Let us assume that $b_{p}$ is the pth AF state at present. $b_{p}$ explores the center position $b_{c}$ and the number $n_{f}$ of its fellow within the visual distance. If $1 \leq n_{f}<P$, and $\Lambda\left(b_{c}\right)>\Lambda\left(b_{p}\right)$, which means state of $b_{c}$ is better than $b_{p}$ and the surroundings is not very crowded, then the $\mathrm{AF}$ moves from $b_{p}$ to $b_{c}$.otherwise executes the behavior of searching food.

\subsection{The behavior of searching food}

Let us assume that $b_{p}$ is the pth AF state at present. The AF selects a state $b_{j}$ randomly within the visual distance(Yang Yu, Ya-fei Tian, Zhi-feng Yin, 2005). If $\Lambda\left(b_{c}\right)>\Lambda\left(b_{p}\right)$, which means the state of $b_{j}$ is better than $b_{p}$, the $\mathrm{AF}$ move from $b_{p}$ to $b_{j}$; otherwise select a state $b_{j}$ randomly again and justify if it satisfy the forward requirement. If it can not meet the requirement after $T N$ times, select a state randomly.

Evaluate the scalar fitness value of each AF by computing the fitness of the equation (5), and compare with the fitness value of $A F_{g}$. If its fitness is higher than the fitness value of $A F_{g}$, instead the $A F_{g}$ by its state; Otherwise the $A F_{g}$ remains unchanged. We identify the lowest merit $\mathrm{K}$ bit $\mathrm{AF}$ in the population and replace it with $A F_{g}$ under elitism. This will ensure that the highest merit AF is propagated throughout the evolution process.

The AFSA terminates after Y-1 number of generations. The AF corresponding to the highest scalar fitness value in (5) is the detected $\mathrm{K}$ number of users' bit vector.

\subsection{Complexity Issues}

The number of computations multiplications and additions required to detect $\mathrm{K}$ bits for the conventional multi-user detector is $2 K N$; The number of computations for the decorrelator is $2 K(K+N)+I N V$ ( $\mathrm{S}$. Verdu. 1986), where $I N V$ denotes computation for the inverse correlation matrix, is on the order of $K^{3}$; The number of computations for the proposed AFSA based detector is $[2 K(K+N)+5 K+1+T N] P Y$, where $T N$ denotes the time of selection a state randomly. Hence, the values of $\mathrm{P}, \mathrm{Y}$ and $T N$ can be adaptively selected, in order to find a tradeoff between the computational complexity and the performance.

\section{Simulations Result}

In system we have adopted the following parameters: the spread sequence are selected as pseudo-noise(PN) with processing gain $N=32$; the receiver, consists of two antennas separated spatially the number of active synchronous users is $K=16$; two-paths slow Rayleigh channels with the second ray delay $T_{c}$ from the first . Perfect power control and CIR estimation was assumed. The strategy based on the sum of the figures of merit from both antennas, will be denoted as S1, while the strategy based on the Pareto optimality will be denoted as S2.

Fig. 3 shows the BER performance against the average signal-to-noise ratio (SNR) for the ASFA-based multi-user detector employing AF-selection strategy S1 and S2.For the sake of comparison, the BER performance of a decorrelator and conventional multi-user detector is also shown. An error floor is observed for the results shown in the figure. This is due to the limitations of the ASFA associated with the particular set of $\mathrm{P}$ and $\mathrm{Y}$ values, not due to the multiple access interference (MAI). It is seen in Fig. 3 that the BER performance for improved when number of generations size was 
increased from 10 to 20 .However, this also increased the computational complexity. Hence, the value of $\mathrm{Y}$ can be selected, in order to find a tradeoff between computational complexity and performance. More importantly, we also see from Fig. 3 that the ASFA employing S2 performs better, exhibiting a lower error floor than S1.

Fig. 4 shows the BER performance against the average signal-to-noise ratio (SNR) for the ASFA-based multi-user detector. It is seen in Fig. 4 that the BER performance for improved when population size was increased from 10 to 20 . However, this also increased the computational complexity. Hence, the value of $\mathrm{Y}$ can be selected, in order to find a tradeoff between computational complexity and performance. We also see from Fig. 4 that the ASFA employing S2 performs better, exhibiting a lower error floor than S1.

Fig. 5 shows the BER performance against the average signal-to-noise ratio (SNR) for the ASFA-based multi-user detector. It is seen in Fig. 5 that the BER performance for improved when the times of searching food $T N$ was increased from 2 to 6 . However, this also increased the computational complexity. Hence, the value of $T N$ can be selected, in order to find a tradeoff between computational complexity and performance. We also see from Fig. 5 that the ASFA employing S2 performs better, exhibiting a lower error floor than S1.

\section{Conclusions}

AFSA is a new kind of intelligence optimization algorithm. Considering the MUD from a combinatorial optimization viewpoint, many scholars have employed the intelligence optimization algorithm such as GA and PSO to solve this problem. We developed a suboptimal multi-user detector based on AFSA. To mitigate the effects of fading, dual-antenna diversity techniques were used. We have shown that AFSA employing the strategy based on the Pareto optimality always exhibit a lower BER compared to those employing the convention strategy. We have also shown that the BER performance can be improved by increasing, number of generations size, the population size and the times of searching food. Hence, the value of Y, P and $T N$ can be selected, in order to find a tradeoff between computational complexity and performance.

\section{References}

Abrao, T. Ciriaco, F. de Oliveira, L.D. Jeszensky, P.J.E. (2006). Particle Swarm and Quantum Particle Swarm Optimization Applied to DS/CDMA Multiuser Detection in Flat Rayleigh Channels. Page(s):133 - 137 Aug. 2006.

B. Steiner. (1997). Uplink performance of a multicarrier-CDMA mobile radio system concept[C]. IEEE Proc of VTC97, 3:1902-1906.

C. Ergun, K. Hacioglu. (2000). "Multiuser detection usiing a genetic algorithm in CDMA communications systems," IEEE Trans. Commun., vol. 48, pp. 1374-1383, Aug. 2000.

D.E. Goldberg. (1989). Genetic Algorithms in Search, Optimization, and Machine Learning[M]. MA: Addison-Wesley.

E. Zitzler, L. Thiele. (1999). Multiobjective evolutionary algorithms: a comparative case study and the strength Pareto approach. IEEE Trans Evol Compute, 3(4): 257-271.

G. Syswerda. (1989). "Uniform crossover in genetic algorithms," in Proc. 3rd Int. Conf. Genetic Algorithms, J. D. Schaffer, Ed.. San Mateo, CA.

Jianmei Xiao; Xiaoming Zheng; Xihuai Wang; Youfang Huang. (2006). A Modified Artificial Fish-Swarm Algorithm. Volume 1, Page(s):3456 - 3460 .

K. Yen, L. Hanzo. (2003). Antenna-diversity-assisted genetic-algorithm-based multiuser detection schemes for synchronous CDMA systems. IEEE Trans Commun.

S. Verdu. (1986). Minimum probability of error for asynchronuous Gaussian multiple-access channels. IEEE Trans Inform Theory, 32(1):85-96.

U. Fawer, B. Aazhang. (1995). A multiuser receiver for code-division multiple-access communications over multipath channels[J]. IEEE Trans Commun, 1995, 43(2): 1556-1565. Feb.-Apr. 1995.

Y. Du, K.T. Chan. (2003). Feasibility of applying genetic algorithms in space-time block coding multiuser detection systems[C]. Proc of ICWOC.

Yang Yu, Ya-fei Tian, Zhi-feng Yin. (2005). Multiuser Detector Based on Adaptive Artificial Fish School Algorithm. China.

Ying Zhao; Junli Zheng. (2004). Particle swarm optimization algorithm in signal detection and blind extraction. 10-12, Page(s):37 - 41, May 2004.

Z. Li, M. J. Juntti, M. Latva-aho. (2005). "Genetic algorithm based frequency domain multiuser detection for MC-CDMA systems," IEEE Proc of VTC2005, vol. 2, pp. 983-987, June 2005. 


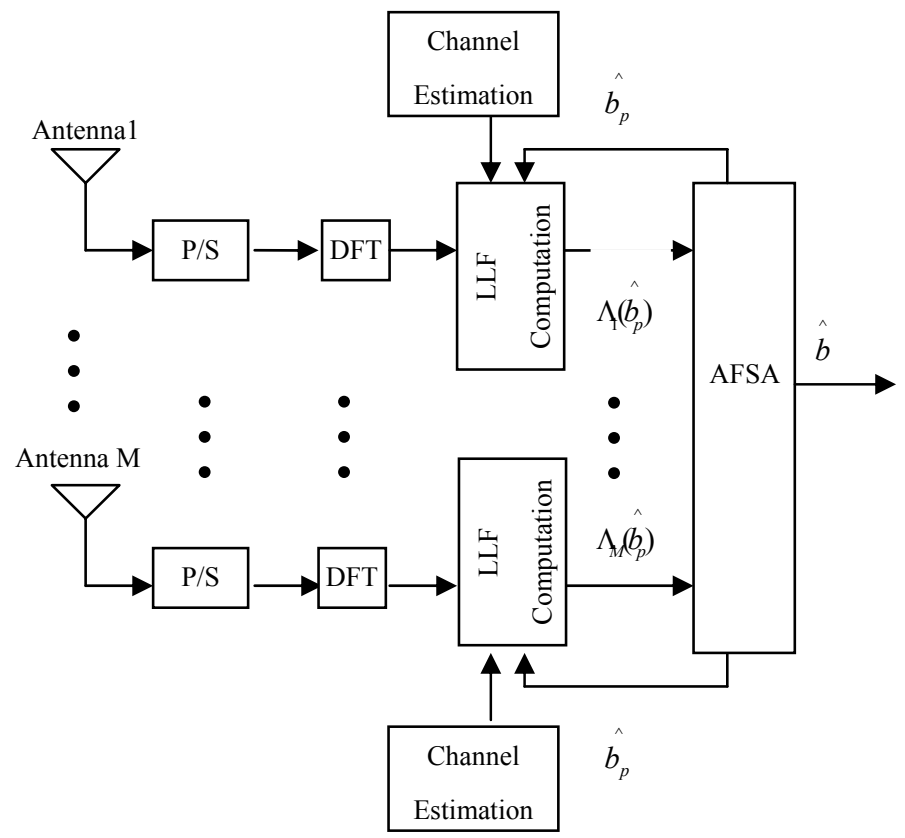

Figure 1. Block diagram of the receiver model

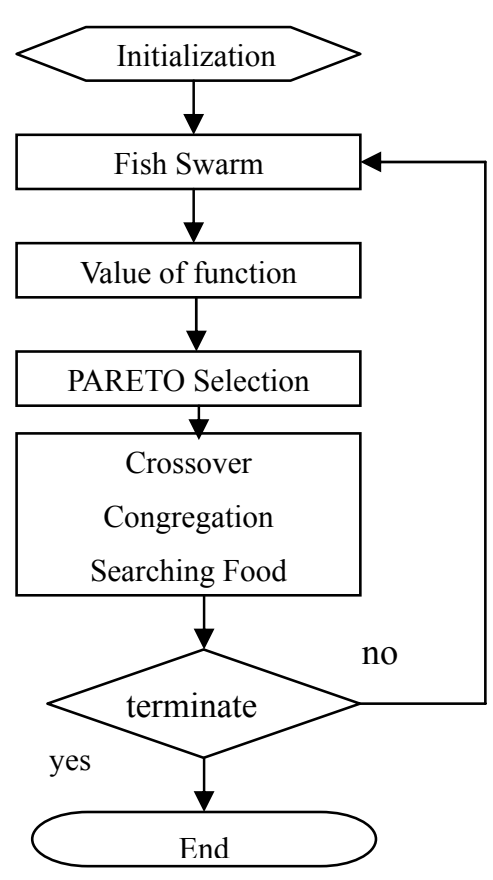

Figure 2. AFSA Program 


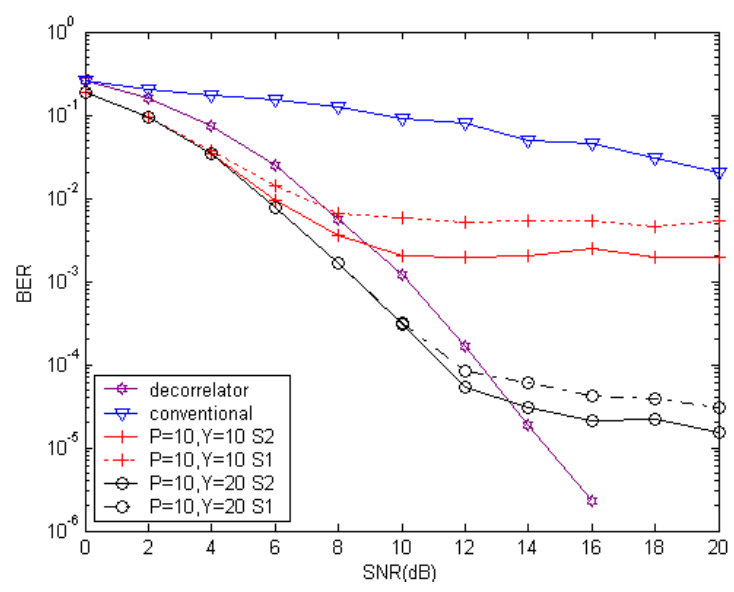

Figure 3. BER performance of the AFSA-based multi-user detector employing the AF-selection strategies of S1 and S2 with population sizes of $\mathrm{P}=10, \mathrm{Y}=10 ; 20 . T N=2$, using signature sequences of length 32and supporting $\mathrm{K}=16$.

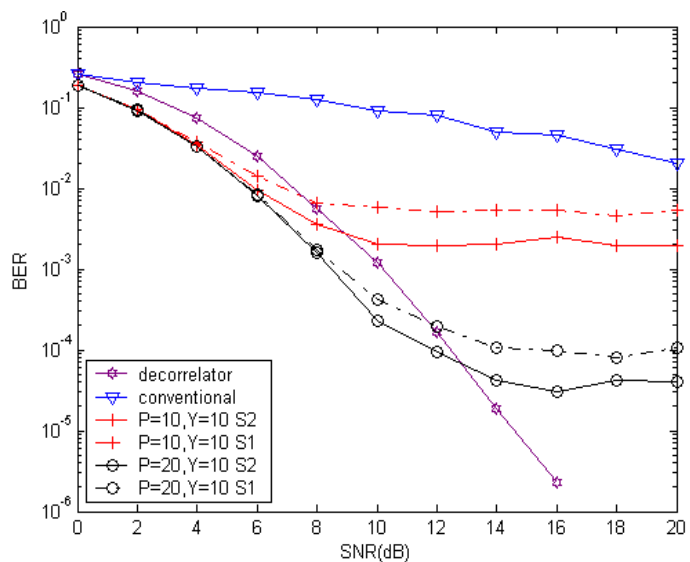

Figure 4. BER performance or the ArSA-based mult1-user detector employing the AF-selection strategies of S1 and S2 with population sizes of $\mathrm{Y}=10, \mathrm{P}=10 ; 20 . T N=2$, using signature sequences of length 32and supporting $\mathrm{K}=16$.

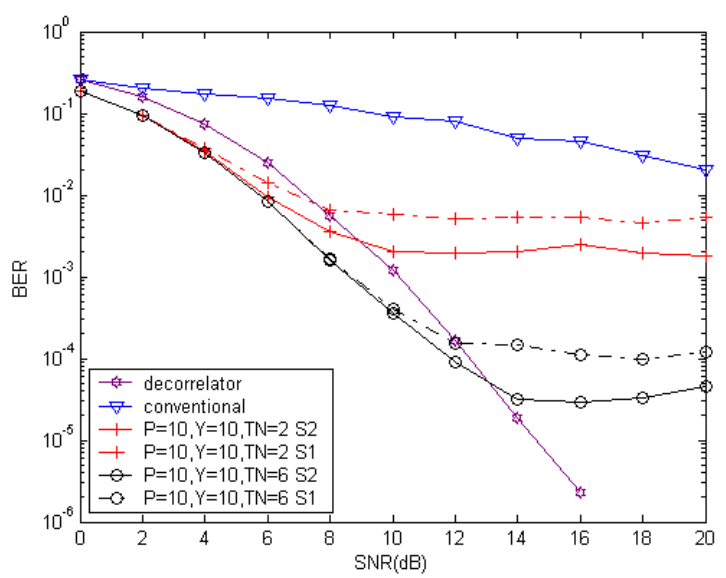

Figure 5. BER performance of the AFSA-based multi-user detector employing the AF-selection strategies of S1 and S2 with population sizes of $\mathrm{Y}=10, \mathrm{P}=10, \mathrm{TN}=2 ; 6$, using signature sequences of length 32and supporting $\mathrm{K}=16$. 